\title{
Alimentation et dessiccation en contexte saharien
}

Le goût du sec

\section{Alimentation and Dessication in the Saharan Context}

The Taste of the Dry

Marie-Luce Gélard

Résumé : Ce texte propose d'aborder la consommation des produits secs et/ou séchés dans une perspective de valorisation de la dessiccation comme norme du "bon ». Les nourritures séchées sont aussi celles qui peuvent circuler dans le contexte migratoire intra et extranational permettant le partage au travers de la commensalité des absents. Elles permettent d'établir une nette distinction entre nourritures humaines et nourritures démoniaques. Enfin, elles seules possèdent des pouvoirs de guérison dans l'univers des rituels thérapeutiques liés à l'alimentation.

Mots-Clés : dessiccation, goût, nourriture, Sahara, saveur

Abstract: This text touches on the consumption of dry or dried products from the point of view of valorization and dessication as a norm of the "good". Dried foods are also those which can circulate in the intra- and extranational migratory contexts thus allowing the commonality of sharing in absence. They also allow us to establish a clear distinction between human foods and demonic foods. And at last, they are the only ones to possess healing powers in the universe of therapeutic rituals linked to alimentation.

Keywords: dessication, flavour, food, Sahara, taste 
Pour le sens commun, le Sahara n'est bon quà être contemplé. Il ne ferait pas bon y vivre. J'ai pu constater à quel point la divergence des points de vue était frappante entre une région présentée par des lectures exogènes (récits de voyages, descriptions des géographes, reportages, témoignages, etc. $\left.{ }^{1}\right)$ comme particulièrement hostile - aride, vide, infertile et inhospitalière - et la vision endogène des populations, pour qui les normes du chaud et du sec se trouvent au cœur de perceptions salvatrices. L'aridité, la sécheresse et la chaleur sont l'objet d'une survalorisation dans les pratiques quotidiennes (alimentaires mais aussi curatives, esthétiques ${ }^{2}$, sociales et culturelles). Je limiterai ici mon propos à l'importance de la dessiccation au niveau de l'alimentation, celles des hommes, mais aussi celle des animaux ${ }^{3}$.

La question des préférences gustatives énoncées par les Aït Khebbach ${ }^{4}$ est itérative et rejoint des pratiques alimentaires au sein desquelles le " sec » est pensé et perçu comme une valeur éminemment positive. La dessiccation préalable de certains aliments suivie de leur réhydratation avant leur consommation est une intervention essentielle à l'esthétique gustative. Si le goût a longtemps été négligé par les Food studies (Von Hoffman 2013), j’ai pour ma part entrepris cette recherche en abordant les univers sensoriels au sein desquels la saveur des aliments est primordiale. Il ne s'agit nullement d'un sens dévalorisé ou déconsidéré comme ce fut le cas en Europe où il a été longtemps méprisé car associé à la gourmandise et à la gloutonnerie, un vice rappelant aussi les images négatives qui lui sont associées : les ogres, les démons, les orgies (Von Hoffman 2013).

Bien manger c'est surtout manger des aliments qui ont du goût ${ }^{5}$ et les qualités gustatives liées à l'environnement saharien (le sec et le chaud). Comme dans toutes les cultures, l'alimentation ne se limite pas à une fonction nourricière. Elle contribue aussi à façonner l'identité et les systèmes symboliques des sociétés. De la sorte, létude attentive des préférences (culinaires, gustatives et esthétiques) permet une meilleure connaissance des groupes.

La recherche présentée dans ce texte fait suite à de nombreux séjours dans le sud-est marocain depuis la réalisation d'un doctorat d'anthropologie il y a près de vingt ans. J'ai poursuivi chaque année mes études au sein du village de Merzouga et des hameaux alentours. C'est donc une ethnographie conduite sur la longue durée qui m’a permis de questionner ces perceptions relatives à l'alimentation.

A la lisière du Sahara, le Sud-Est marocain désigne la région située au sud de la ville d'Er-Rachidia et à l'est de celle de Zagora. Elle comprend l'extrémité orientale de l'Anti-Atlas, à l'est le Hamada du Guir et au sud Agoult et Tabelbala A l'est du village de Merzouga, les dunes de sable de l'erg Chebbi sétendent sur une surface de 22 kilomètres de long sur 5 kilomètres de large. Du point de vue climatique, le Sud-Est marocain et les territoires algériens qui le jouxtent à lest appartiennent au domaine saharien, avec de faibles précipitations - autour de 60 millimètres par an (70 $\mathrm{mm}$ à Béchar, $59 \mathrm{~mm}$ à Merzouga) voire moins (30 mm à Beni Abbès). Elles sont nettement au-dessous des 100 millimètres 


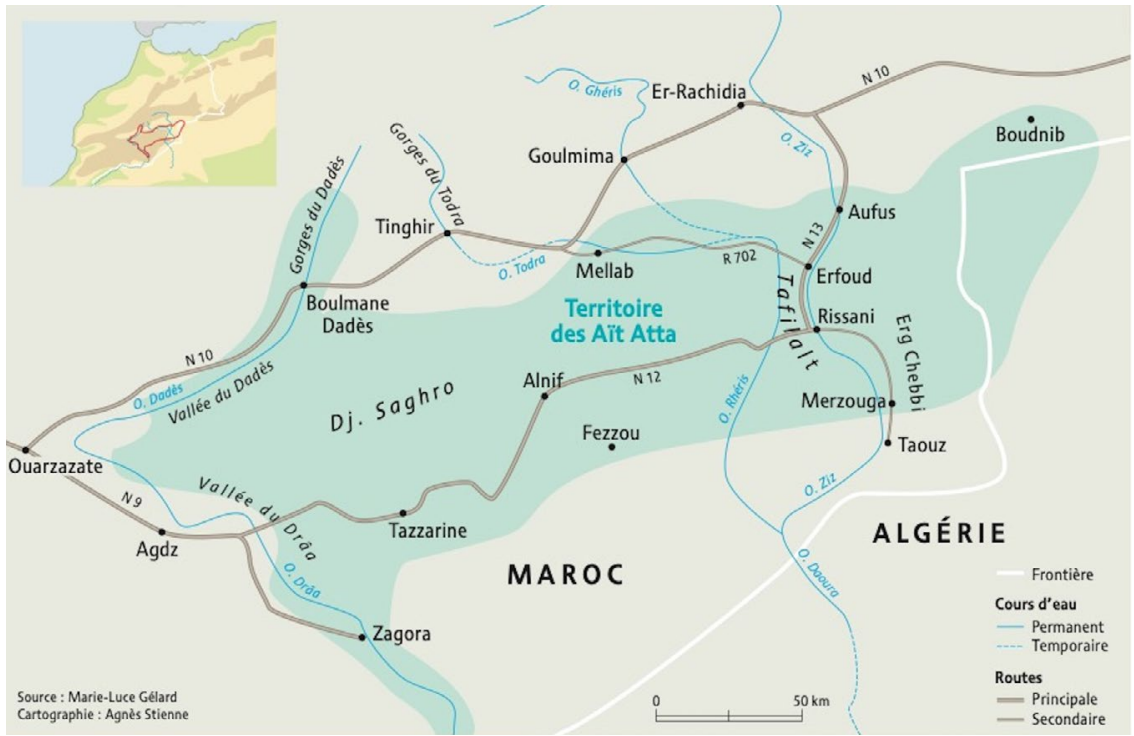

Carte 1 : Localisation géographique des Aït Atta et du village de Merzouga. (C) M.-L. Gélard, Merzouga, 2020.

que l'on retient par convention comme « limite » saharienne. Les températures moyennes minimales et maximales mensuelles à Merzouga sont, selon deux sites Web, pour le premier (Climate-Data.org) respectivement de $25,6^{\circ} \mathrm{C}$ (température minimale) et de $43,2^{\circ} \mathrm{C}$ (température maximale) pour le mois le plus chaud, juillet, et, pour le second site (Meteoblue.com), toujours pour juillet, $22^{\circ} \mathrm{C}$ et $41^{\circ} \mathrm{C}$. Notons que le caractère saharien des températures règne assez loin au nord de Merzouga. En été, des pointes de température dépassent régulièrement les $50^{\circ} \mathrm{C}$. Ces précisions climatiques sont importantes car lélément le plus notable concerne la sécheresse qui distingue, mieux que la chaleur, les mois d'été des autres mois. Au Sahara, l'air est particulièrement sec (le degré hygrométrique est de 20 à 30 pour cent en été et de 50 à 60 pour cent en hiver) et c'est cette caractéristique qui rend supportable les températures élevées.

Le Sud-Est (Tafilalt) a été longtemps l'une des principales portes du Sahara, lieu déchanges entre le Nord méditerranéen et le Sud africain. Cette région est le lieu de naissance de l'actuelle dynastie alaouite.

Partiellement sédentarisés dans la région, les Aït Khebbach appartiennent à la confédération tribale amazighophone ${ }^{6}$ des Aït Atta. Ils en constituent la frange la plus saharienne ${ }^{7}$. Ils furent longtemps les guides des routes caravanières menant de Sijilmassa au Bilad al-Sudan, soit l'actuel Mali. Leur sédentarisation contrainte au début du vingtième siècle n’a pas été totale, ils sont aujourd'hui encore parmi les derniers groupes marocains qui nomadisent. Certaines familles se partagent entre la vie sous la tente, la pratique de lélevage 
(moutons et dromadaires) et une habitation sédentaire. Ainsi, le nomadisme et les activités pastorales associées perdurent en complément d’autres activités professionnelles sédentaires. On peut être à fois nomade/éleveur, pratiquer une activité agricole oasienne au village et posséder une auberge à des fins touristiques. Contrairement à la fin annoncée du nomadisme, on assiste, au Maroc, à l'essor de systèmes économiques multiples, où le pastoralisme devient une activité parmi d’autres (Skounti 2012).

\section{La dessiccation alimentaire comme norme du «bon »}

Le séchage des denrées alimentaires a longtemps été utilisé, en contexte saharien et ailleurs, comme un procédé de conservation. C'est encore le cas de la viande et des abats, mais aussi de certains fruits et légumes (mulghiya, ail, dattes). Lapparition et la diffusion à l'ensemble des foyers d'appareils frigorifiques n’a pas changé les pratiques car les aliments sont aussi séchés pour des raisons culturelles, celles précisément liées au goût. La congélation de la viande est assez peu fréquente, en principe elle est achetée le matin pour une consommation dans la journée.

La dessiccation est un procédé indispensable dans l'utilisation des simples, un des "éléments qui fait acquérir à une plante le statut de "médicinale" " (Garetta 2007 : 262). Dans le cas saharien, le séchage des nourritures s'inscrit dans cette même perspective identitaire où le goût de certains aliments est indissociable de leur dessiccation.

Ainsi, les mulghiya (Abelmoschus esculentus L.) sont des fruits très appréciés. Or, contrairement à d'autres usages en Afrique de l'Ouest notamment (où il est nommé gombo), les mulghiya sont surtout consommés séchés puis réhydratés. Ils sont alors considérés comme meilleurs, parce que plus goûteux. Ce fruit, utilisé en fait comme un légume, contient une substance mucilagineuse qui sert d'épaississant. Laspect gélatineux et de ce fait humide, ne fait pas partie des préférences culinaires dans le Sud-Est marocain, il est sans doute séché pour cette raison. Il s'agit du fruit d'un Hibiscus que l'on retrouve dans les régions tropicales et subtropicales, c'est pourquoi il s'est bien implanté dans les oasis et les palmeraies, du fait de la chaleur et de l'irrigation.

D’une manière générale, toutes les productions des palmeraies du village ${ }^{8}$ sont valorisées, l'ail (très apprécié) et les piments - qui sont de très petites tailles du fait du manque d'eau - sont considérés meilleurs parce que plus " concentrés ». Dans la ville voisine de Rissani, on trouve aujourd'hui de l'ail en provenance de Chine, dont les gousses sont larges de 1 à $2 \mathrm{~cm}$, contre quelques millimètres pour celui des jardins. Seul celui de l'oasis est considéré comme ayant du goût, l'ail chinois est trop « mouillé ». L'ail est aussi séché et mélangé à du sel pour servir de condiment dans la préparation des plats.

La consommation hebdomadaire ${ }^{9}$ de couscous donne lieu à des échanges de nourriture entre maisonnées ${ }^{10}$ mais répond surtout à des critères de 
préparation très spécifiques à la région. En effet, si partout ailleurs le couscous se compose de la semoule de blé dur agrégée et de sauce (dont la composition est variable), à Merzouga le plat de couscous est servi avec la semoule très légèrement imbibée de sauce, la graine est arrosée afin de ne jamais laisser de jus dans le fond du plat (Fig. 1). Le couscous ne doit pas être "mouillé » ni surtout "noyé » de sauce ${ }^{11}$. Dans la région, le couscous est aussi parfumé d'un lichen séché (l'évernie ou " mousse du chêne ») (Fig. 2), le parfum est obtenu par la fumée qui se dégage du lichens brûlé. Là encore, c'est à la dessiccation qu'il est fait allusion, d'une part parce qu'on utilise un lichen séché et, d'autre part, qu'on le brûle afin que le parfum se répande via la fumée (dont on sait aussi les vertus astringentes). Ce parfum alimentaire est très apprécié.

La soupe d’orge concassée est consommée le soir, agrémentée de navet l'hiver, elle est très épaisse et s'apparente à une sorte de bouillie, une soupe trop liquide est dépréciée. Elle est souvent épaissie de farine de blé. C’est la consistance de la «soupe » qui en change la nature même, se nourrir nécessite de manger un aliment épais, nourrissant au sens de l'encombrement qu'il génère dans l'estomac. Le liquide ne peut satisfaire cette exigence.

La consommation de dattes est aussi une alimentation appréciée et l'objet d'une grande attention dans leur conservation. Lors de la récolte, les dattes sont conservées tassées fortement dans des sacs et séchées sur les toits en terrasse. Elles sont alors agglomérées les unes aux autres sous la forme d'une pâte épaisse à laquelle on ajoute parfois des herbes séchées qui la parfument. Il s'agit d'un fruit dont la texture sèche et la saveur très sucrée est valorisée. Il est surtout consommé avec du thé chaud.

La nourriture carnée que l'on sèche le plus couramment est constituée des entrailles du moutons (panses, intestins et estomac) appelées corda ${ }^{12}$. Ces abats ne sont quasiment ${ }^{13}$ jamais mangés frais, ils sont séchés durant plusieurs jours. Le séchage nécessite tout d'abord une préparation des abats (Fig. 3) qui sont salés et pimentés puis découpés en fine lanières et attachés ensemble à l'aide d'un morceau d'intestin grêle. Ils sont ensuite séchés sur un fil durant plusieurs jours (Fig. 4). Leur conservation peut alors durer plusieurs mois ${ }^{14}$. On procède de la même manière avec des morceaux de viande. Au niveau gustatif, les viandes séchées ont un goût plus fort et particulier que l'on pourrait opposer à un aliment fade. Elles sont très prisées.

Les perceptions du goût et ici du " bon goût » s'associent aux bienfaits de la dessiccation, intimement liée à une représentation de la nature où le sec et le chaud apparaissent comme les normes du bien-être corporel. Ainsi, l'humidité, celle du climat notamment est fortement dévalorisée, elle serait responsable des maux du corps dont souffrent les urbains. J'ai montré ailleurs (Gélard 2016) comment la chaleur était instrumentalisée à des fins thérapeutiques par l'utilisation des «bains de sable ». Il s'agit, par l'immersion du corps dans le sable brûlant aux heures les plus chaudes, de guérir différents maux : rhumatismes, douleurs articulaires, paralysie, etc. Le procédé doit s'effectuer durant plusieurs jours consécutifs, aussi la pratique s'apparente-elle à une cure. 


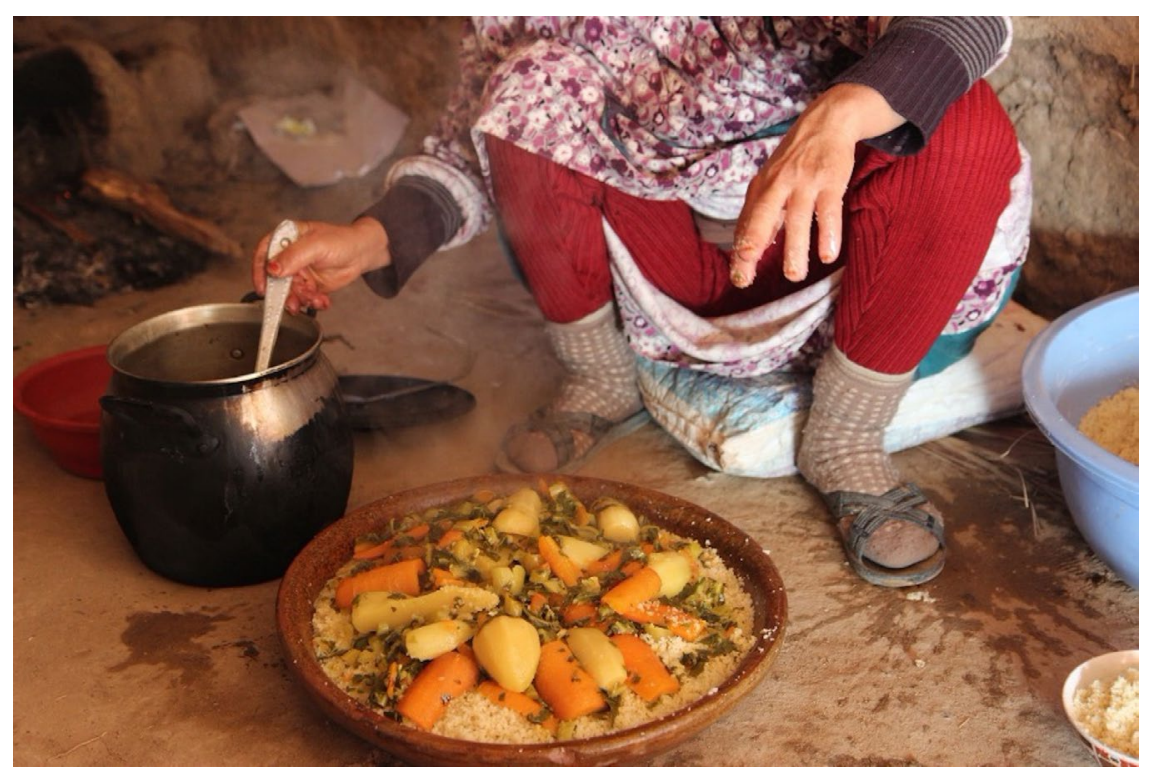

Figure 1 : Plat de couscous prêt à servir et imbibé de sauce entièrement absorbée par les grains. (c) M.-L. Gélard, Merzouga, 2016.

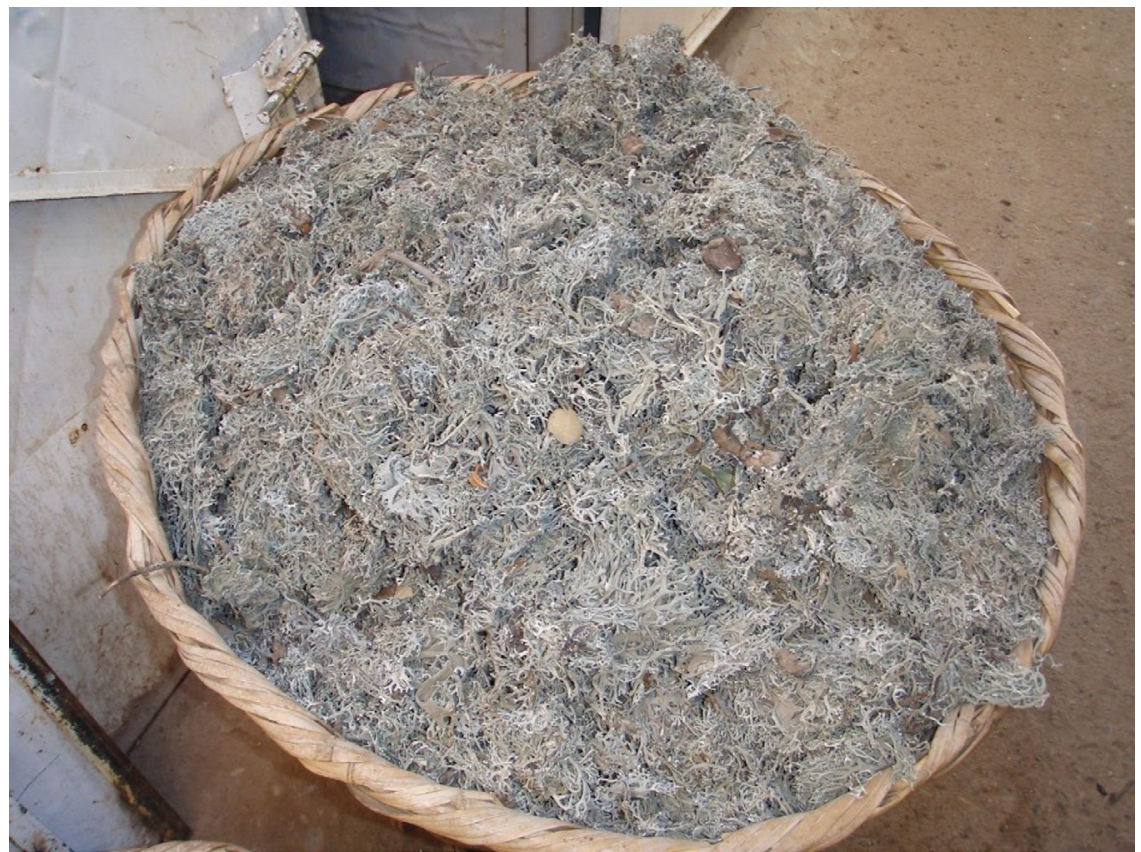

Figure 2 : Lichens séchés, marché de Rissani. @ M.-L. Gélard, Merzouga, 2009. 


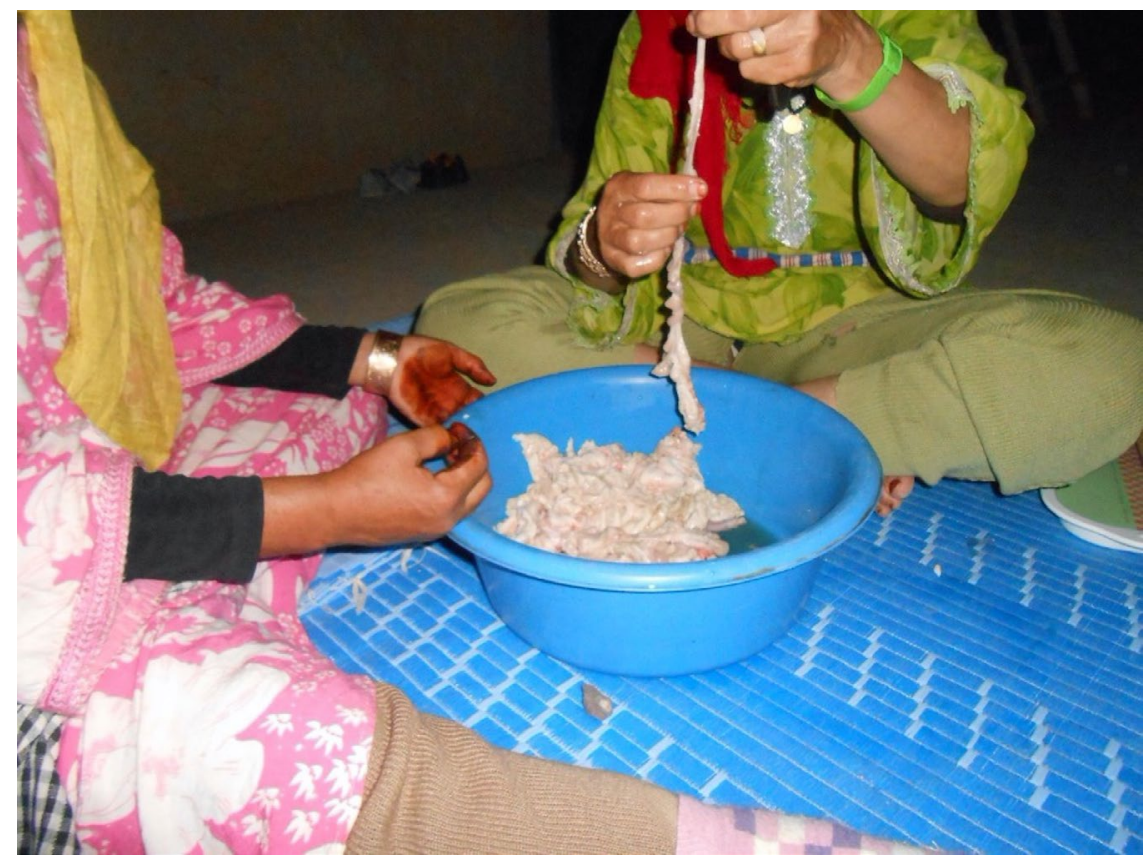

Figure 3 : Préparation de corda. () M.-L. Gélard, Merzouga, Takucht, 2012.

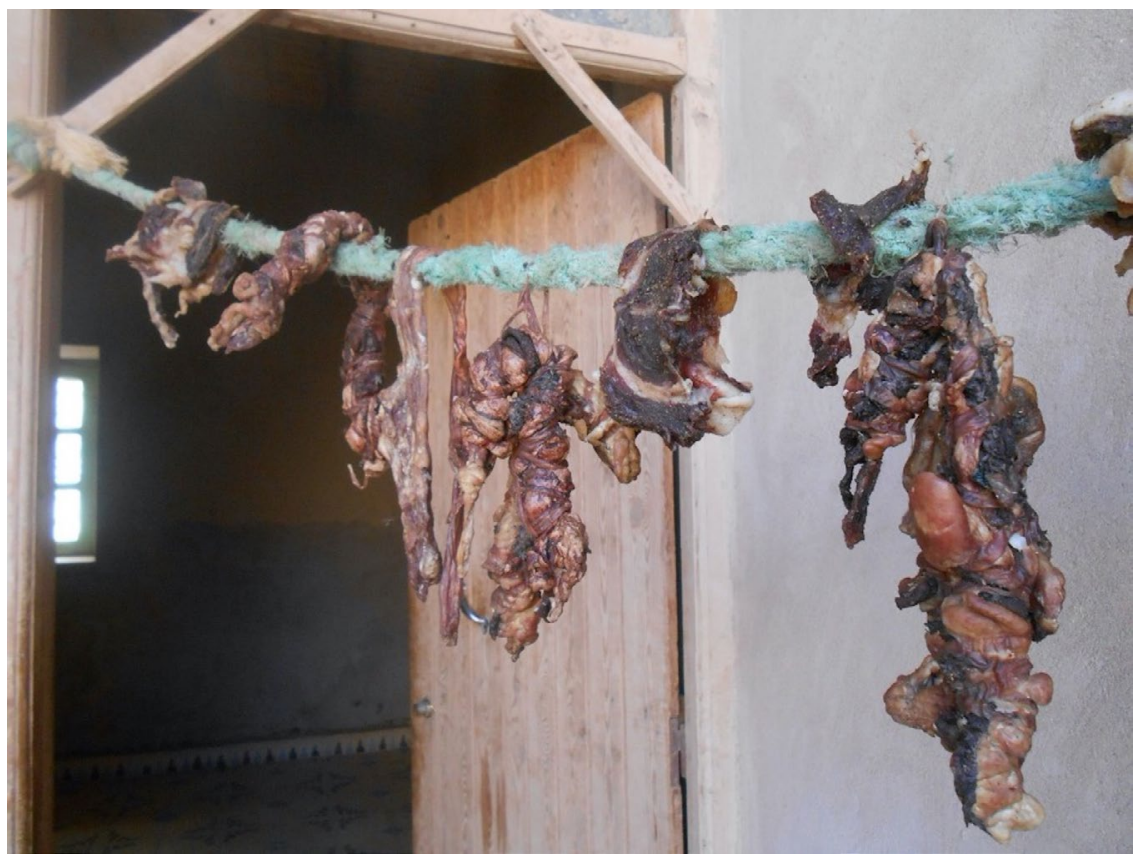

Figure 4 : Viande et corda qui sèchent. (c) M.-L. Gélard, Merzouga, 2012. 
Cette forme de tourisme endogène est en pleine expansion, elle permet une appropriation positive de l'environnement saharien, longtemps et encore marginalisé, par rapport au monde urbain ${ }^{15}$.

Lopposition entre des nourritures perçues comme " chaudes » ou « froides » est un poncif au sein de nombreuses cultures, comme ici en contexte amazighophone (Hincker 2005 ; Figuereido 2001). On est très proche du système «hippocratique» mais ce qui semble moins documenté par les études concerne cette valorisation, survalorisation de la dessiccation ${ }^{16}$.

Les nourritures séchées permettent aussi leur transport. Mulghiya, corda, viande de l'Aïd et gâteaux secs sont les principales nourritures qui circulent en contexte migratoire, dans tout le Maroc mais aussi en Europe. Leur dessiccation permet ce transport et une conservation parfaite. Au sein de la migration extra-nationale, la dessiccation apparaît comme une opportunité très importante de faire voyager les nourritures du pays d'origine. Il est ainsi possible de conserver la viande des absents, dans une société où le partage et l'équité alimentaire sont primordiaux. De la sorte, la viande de l'Aid (fête du sacrifice musulman) en étant séchée peut être envoyée aux absents, lesquels bénéficieront ainsi des bienfaits de la commensalité religieuse partagée et des effets bénéfiques (baraka) qui lui sont attribués. Le sacrifice commémore celui d'Ibrahim et doit en principe être réalisé par tout homme marié ; aux fondements religieux sajoutent aussi des fondements identitaires (Brissebarre 2017 : 618). Aujourd'hui, la difficulté inhérente à la pratique du sacrifice sanglant en contexte migratoire (notamment européen) rend parfois impossible sa réalisation, la circulation de la viande de l'Aïd, sous sa forme séchée, apparaît pour beaucoup comme une compensation, certes très insuffisante mais une sorte d'alternative permettant d'éviter une période sans sacrifice aucun...

La viande du sacrifice de l'Aïd el-Kébir est donc l'une des nourritures les plus prestigieuses comme en témoigne le soin constant qui lui est porté. Il sagit en premier lieu de lélevage de l'animal ou de son choix - lors d'un achat - et de lattention à son bien-être jusquau moment du sacrifice ${ }^{17}$, de la découpe de la carcasse, de l'entrée de la viande dans la maison, etc. et, en dernier lieu, d'un rituel où la dessiccation est à nouveau mise en exergue. Dans la région, le rituel d'isgar consiste à recueillir certains des os du sacrifice : mâchoires, omoplate droite, pattes et tête. Ces os sont conservés et on prend soin lors de la découpe de ne pas les briser. Ce sont les femmes qui se chargent de récupérer lesdits os auxquels elles mélangent du cumin, du piment rouge, de la cannelle, du henné et des œufs. Dans ce rituel, on n'utilise que des aliments réputés chauds et en lien avec la fécondité (œuf et henné). Ce sont ensuite les jeunes filles célibataires qui se parent comme pour un jour de fête et emportent les os vers un tamaris à l'extérieur du village. Durant leur parcours elles chantent « on va jeter l'isgar ${ }^{18}$ et le mal ne reviendra plus ». Les os sont déposés à terre puis suspendus à l'arbre, le vent agite les os et c'est ainsi qu'il emporte tous les maux de la famille. Le rituel agit aussi comme une offrande aux jnûn : pour sattirer leur bienveillance, on leur offre les os comme pendant du sacrifice ${ }^{19}$, mais ici un sacrifice non-sanglant. 
D’une manière générale, l'appréhension très positive de la dessiccation alimentaire ne se limite pas à la nourriture humaine, elle trouve aussi une résonance importante dans le choix des aliments que l'on donne aux animaux.

\section{La nourriture quotidienne des moutons : l'obligation de la dessiccation}

Lélevage de quelques têtes de bétail se pratique dans la plupart des familles pour répondre au besoin en nourriture carnée à l'occasion de différentes fêtes (Aïd, tasmiya ${ }^{20}$, retour des proches, etc.). Ces moutons, appelés « moutons de case ", désigne des moutons élevés dans l'habitation familiale en milieu rural subsaharien ; on parle aussi de "mouton de ville » (Brisebarre et Kuczynski 2009: 66).

Ce sont en majorité les femmes qui soccupent de nourrir les quelques moutons et chèvres qui vivent dans de petites bergeries jouxtant les maisons (Fig. 5). La nourriture donnée aux animaux est l'objet d'une grande attention, ainsi on prend toujours soin de ne jamais donner une nourriture qui aurait été en contact avec de la viande. L'épidémie dite de « La vache folle » en Europe est observée avec un profond dégoût par les habitants de Merzouga, donner de la

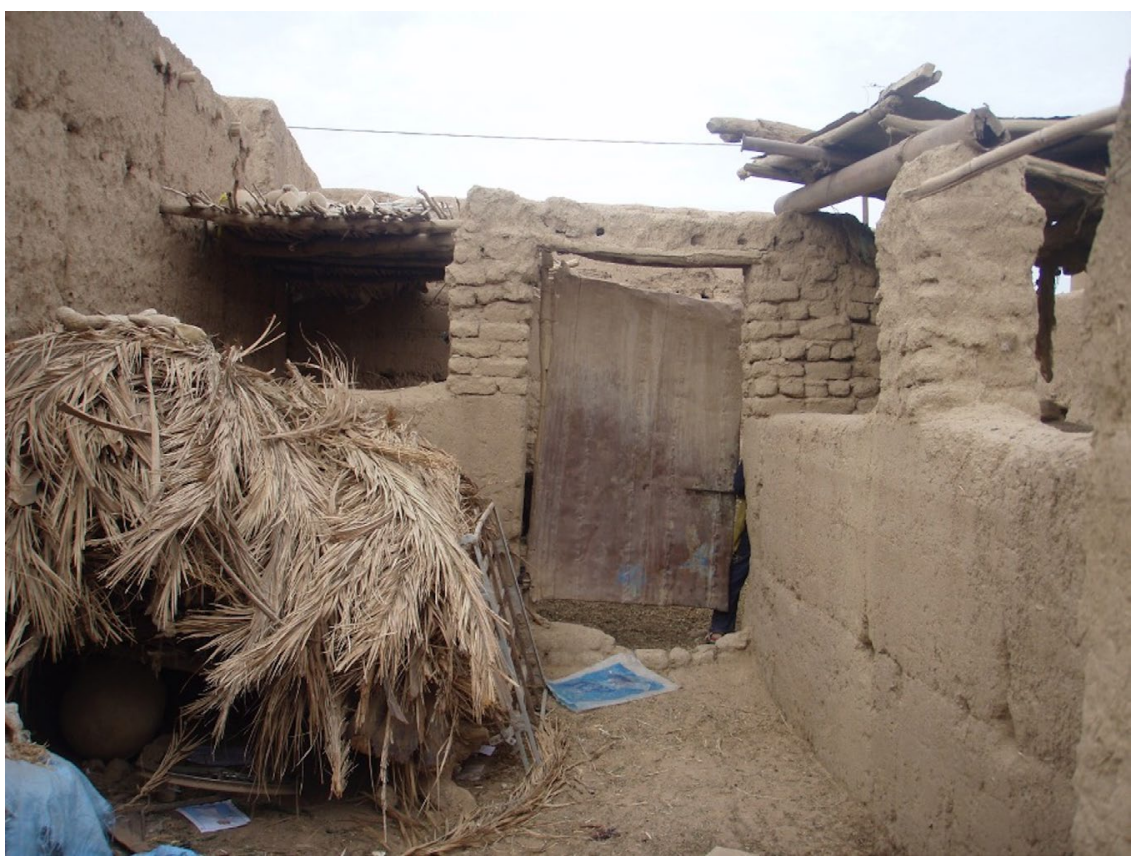

Figure 5 : Bergerie qui jouxte une habitation. @ M.-L. Gélard, Merzouga, 2010. 
viande à des herbivores est considéré comme une aberration et une maltraitance animale grave.

La mise en pâture n'est pas réalisée, ces ovins et caprins ne quittent jamais les lieux. Les conditions climatiques et les sécheresses endémiques rendent difficile la pérennisation d'espaces dédiés aux pâturages. Toute nourriture donnée doit être séchée, elle serait sinon indigeste et nocive pour des animaux qui ne pratiquent aucun exercice et nont en général que quelques mètres carrés d'espace. Les restes alimentaires (épluchures des légumes et des fruits, feuilles de thé, restes de pain, etc.) sont utilisés, car la luzerne cultivée dans la palmeraie du village ne suffit pas à nourrir le bétail. Les écorces des agrumes l'hiver et des pastèques et melon lété (consommées en grande quantité) sont coupées en petits morceaux, séchées au soleil et données au bétail. Une nourriture trop humide et trop fraîche (pleine d'eau) ferait gonfler la panse des ruminants. Il s'agit d'un phénomène très connu des éleveurs : la météorisation.

Depuis peu, on donne à manger aux caprins et ovins des cartons, pratique urbaine très répandue en Mauritanie et au Sénégal ${ }^{21}$. Ces animaux ont la capacité de digérer la cellulose, ce qui permet de fournir la « ration d'encombrement » quotidienne nécessaire en absence de paille et de fourrage en quantité suffisante. Afin de pallier ce manque, on donne aussi aux moutons les noyaux des dattes, produit sec par excellence, préalablement concassés à l'aide d'un pilon de pierre ${ }^{22}$. Toute nourriture est ainsi précieusement mise de côté pour nourrir quotidiennement les quelques têtes de bétail.

En dehors de lélevage familial dont la viande est considérée comme la meilleure, une très nette préférence en matière de choix des animaux et de leur provenance illustre la valorisation des produits secs. Ainsi, les habitants de Merzouga les plus fortunés achètent leurs moutons auprès des nomades. Ces derniers élèvent leur cheptel aux alentours de l'erg Chebbi, où les animaux se nourrissent uniquement de végétaux herbacés secs du désert. La viande issue de ces élevages est non seulement considérée comme meilleure, mais elle est aussi susceptible de guérir certains maux (telles les douleurs d'estomac, les ulcères, les diabètes, le rachitisme, etc.).

Le lait et le thé sont les seuls aliments liquides valorisés. Concernant le thé dont la consommation quotidienne est importante il est, là aussi, évalué en fonction de sa consistance. Un thé clair et juste parfumé de menthe fraîche est réservé à l'usage des touristes, le thé des Merzougui est fort et très sucré. Afin de rendre son aspect encore plus sirupeux, on y ajoute un mélange de plantes et de résines qui le rendent alors mousseux.

Le lait animal ${ }^{23}$ est aujourd'hui peu consommé par les adultes ${ }^{24}$ sauf sous sa forme de lait caillé, donc épais (leben en arabe). Ce qui est le plus apprécié, c'est la consommation du colostrum. Le terme Adghes (/adjes/) est un terme panberbère, usité de la Libye au Maroc et au monde touareg, il signifie « colostrum, premier lait après la naissance »; une stabilité sémantique et formelle qui est déjà intéressante en soi puisqu'elle est un indice de l'importance accordée à cette « première lactation ${ }^{25} »$. De plus, le colostrum tant humain qu'animal ${ }^{26}$ 
est connu de tous (enfants comme adultes), ce qui n'est pas le cas dans d'autres cultures ${ }^{27}$.

Le colostrum est perçu comme une substance plus concentrée que le lait, les propos se réfèrent à une consistance plus épaisse, formant dit-on une sorte de « nœud ». Il est très calorique et destiné à lénfant qui vient de « tomber $^{28}$ » - expression qui désigne la naissance. On dit que le nourrisson doit prendre le colostrum, car c'est là qu'il va puiser sa force. Pour évoquer la bonne santé d'une personne on utilise l'expression " "Tu es fort" car "tu as mangé le colostrum de ta mère" » (information orale, Merzouga 2014).

Le colostrum des ovins et des caprins élevés dans le cadre familial susmentionné est l'objet d'une grande attention. On en prélève toujours une partie, car sa consommation complète par le petit serait néfaste (coliques). Le colostrum est produit pendant trois jours après la mise bas. Les Aït Khebbach apprécient vivement le colostrum animal qui n’est jamais consommé cru ( $a d r s)$, mais cuit $(\text { tichlit })^{29}$. Il a alors la consistance des œufs brouillés, il est salé et consommé avec du pain. Passé les trois premiers jours, il se transforme en lait et ne peut plus être cuit. Ce colostrum animal est une nourriture d'exception. Notons cependant que sa consommation est réservée aux adultes et aux adolescents, car on estime qu'il serait trop fort pour les enfants en bas âge (interdit à ceux qui sont encore allaités ${ }^{30}$ ).

Il reste à évoquer l'importance et le rôle du sel comme élément essentiel à la dessiccation mais aussi à la séparation des univers humain et démoniaque. La dessiccation est toujours liée à la chaleur et au climat saharien qui seul est pensé comme permettant ladite dessiccation.

\section{Nourriture humaine et nourriture démoniaque : le sel comme frontière}

Les saveurs permettent de distinguer les nourritures humaines des nourritures démoniaques. L'expression " mon sel dans ton ventre " se réfère aux liens qui naissent entre deux individus ayant partagé un repas. Le sel attache, ligote et crée une relation, un lien par la commensalité. «Le sel consommé en commun crée une association mystique entre les consommateurs d'un même repas. (...) Le sel dévoile toute personne qui ne respecte pas les obligations morales consécutives au partage du repas. En effet, lorsque deux personnes ou plusieurs absorbent en commun les mêmes aliments, la nature de leurs rapports change ipso facto. Les consommateurs d'un même repas doivent s'abstenir de se faire du mal. Le transgresseur du pacte implicite et mystique ne peut échapper au sel qui a le pouvoir de "ligoter", d'“attacher" " (Rachik $1990: 63$ ).

Les jnûn détestent le sel et toutes les saveurs délicates, aussi la nourriture salée des humains ne peut-elle leur convenir. Le sel permet d'instaurer une séparation nette entre le monde des hommes et celui des démons. De la sorte, le goût humain soppose au fade démoniaque. Au niveau de la langue même, 
on utilise des métaphores pour ne pas avoir à prononcer le mot jnûn, car les nommer, c'est les appeler. Pour se prémunir de leur présence, on dira alors " ceux que le sel nous dissimule " (uidar ar tissentl tisint). Les jnun mènent une vie en parallèle des hommes, dans une sorte de monde inversé, dans les espaces interstitiels (seuils physiques, périodes de passage d'un état à un autre, tels naissance, mariage, mort, etc. $)^{31}$. Le fait de saler la nourriture garantit que les jnûn ne vont pas sen approcher. La proximité des jnûn avec le monde des hommes, notamment dans les habitations, rend nécessaire de nettoyer tous les ustensiles le matin avant de les utiliser. En effet, durant la nuit, ils peuvent semparer des objets et s'approprier les espaces domestiques. C'est aussi la raison pour laquelle toute activité (pétrissage du pain, lessive, filage de la laine, etc.) doit impérativement être précédée de la formule "bismillah » (au nom de Dieu) laquelle permet de les éloigner car ils prolifèrent dans ces moments de passage.

La nourriture est protégée dès qu'elle est salée. On prend ainsi toujours soin de mettre du sel sur le sang des animaux égorgés afin de bien signifier que le sacrifice ne leur est pas destiné (ce qui peut parfois être le cas). Le sacrifice à destination des jnûn est fréquent et permet déviter leurs actions maléfiques : prémisses lors des récoltes, disparition du lait maternel, lieux inhabités que l’on investit, etc. Se prémunir de l'action maléfique et partager ainsi un repas avec les jnûn sous forme doffrandes exige de leur offrir un met (parfois un repas) fade et insipide. Lorsque l'on soupçonne leur intervention, notamment dans le cas de la disparition du lait maternel, la mère allaitante prépare un repas sans sel qu'elle dépose dans un endroit isolé de la maison. Elle se lève la nuit, fait du bruit pour éloigner les jnûn attablés (elle ne peut les voir) et mange un peu du plat en énonçant la formule adaptée signifiant dans "vous mavez pris mon lait, que Dieu garde à chacun son bien ». Ainsi, une sorte de partage de commensalité a lieu, censé rétablir l'harmonie.

Certains des lieux de prédilection des jnûn sont parfumés par fumigation, car ces derniers détestent les « bonnes » odeurs et les saveurs alimentaires.

\section{Nourritures et guérison : le sec et le chaud}

Si la plupart des maux du corps sont soignés par l'utilisation de la chaleur ${ }^{32}$, la nourriture est aussi susceptible de guérir. Là encore, seules les nourritures dites chaudes et/ou échauffantes apportent la guérison. Je limiterai mon propos à deux exemples, celui des céphalées et celui des refroidissements.

Les oppositions structurelles entre le chaud et le froid ne sont pas nécessairement pensées dans une harmonie des contraires, de complémentarité. Ce qui est recherché c'est une continuité des sensations, il convient de ne pas «brutaliser » le corps en mettant en contact le chaud et le froid. Les nourritures dites « froides » n'entrent que très rarement dans les thérapeutiques traditionnelles. Ainsi, la menthe, aliment froid dont l'usage au Maroc est très abondant, est fort peu consommée à Merzouga (hormis dans le cadre touristique) et le thé 
à la menthe est peu apprécié. On lui préfère en toutes saisons un thé parfumé d'herbes dites chaudes (absinthe ou mélange d'herbes sèches ${ }^{33}$ ). De manière identique, l'eau trop fraîche n'est pas consommée, encore moins glacée ${ }^{34}$. L'eau est refroidie dans des jarres de terres dont l'extérieur est humidifié. On utilise aussi des bidons recouverts de tissu, lequel est mouillé afin que, par évaporation au contact de l'air, il refroidisse l'eau de quelques degrés seulement. Boire trop froid lorsqu'il fait chaud crée un contraste trop brusque, une sorte de déséquilibre des températures pensé comme néfaste pour le corps. Tout passage d'un état à un autre (chaud/froid, sec/humide, calme/agitation, etc.) est susceptible aussi d’attirer les jnûn. Ces derniers se déploient, comme évoqués précédemment, dans les espaces interstitiels et dans les moments de passage, mais aussi dans le quotidien. Ainsi, éteindre un feu avec de l'eau est estimé trop brutal : il faut soit le laisser séteindre, soit l'étouffer doucement avec du sable. Se doucher lorsquon est en sueur déséquilibre le corps et peut attirer les mauvais esprits. Il faut donc attendre que le corps se refroidisse lentement. Courir, crier, faire des gestes violents ou manifester bruyamment une émotion est une perte de contrôle de soi qui perturbe l'harmonie du monde.

En cas de céphalées, le remède le plus efficace est celui de la fumigation de cornes de mouton. L’odeur de corne brulée est âcre et forte, elle pénètre les vêtements et c'est à la fois la fumée dessicative et son origine (tête animale) qui permettent de réduire la douleur.

Les rhumes, ou tout refroidissement, nécessitent de consommer une nourriture chaude et sèche, il faut retirer le froid du corps. Pour ce faire, on prépare une boule à l'aide de dattes, de miel et de cumin en poudre. Il convient de l'avaler rapidement et de rester au chaud, sous des couvertures plusieurs heures durant.

Dans le contexte saharien, les bienfaits de la dessiccation sont nombreux et permettent de mieux comprendre une partie des représentations sociales et symboliques et, plus généralement, des préférences culturelles et des tonalités spécifiques de l'environnement - un environnement salvateur par excellence si l'on sait y être attentif. De nombreuses histoires rapportent de quelles manières le désert peut sauver les hommes. C’est le cas du célèbre récit fondateur de Tin-Hînan (littéralement celle de la tente) : cette femme qui, venue du Tafilalt pour s'installer en Ahaggar (Sud-Est algérien), ne survécut que grâce à l'habileté de sa servante qui recueillit des graines amassées par les fourmis (du genre messor) capables d'emmagasiner plusieurs litres de graines dans leur fourmilière. Le Sahara n'est donc pas un espace vide : l'on peut même y puiser des ressources alimentaires insoupçonnées, mais cela suppose de le connaître intimement.

Lincidence environnementale sur les pratiques, notamment alimentaires, souligne bien la survalorisation d'une nature bienfaisante. Le Sahara est bon à vivre, l'adéquation homme/plante/univers est poussée à son paroxysme, au point que, selon beaucoup des Aït Khebbach, seuls la région et son climat sec et chaud permettent une véritable harmonie des corps. 
Marie-Luce Gélard est anthropologue spécialiste des sociétés amazighophones sahariennes (Maroc, Algérie) et de la parenté de lait. Ses recherches sorientent autour des modalités de la perception sensorielle et de leurs codifications corporelles par la mise en place d'une anthropologie des sens. Dernier ouvrage publié en 2017, Les sens en mots, Paris, Pétra.

Email : mlgelard@yahoo.fr

Marie-Luce Gélard is an anthropologist specialised in Saharian, Amazighspeaking societies (Morocco, Algeria) and in milk parenthood. Her research is directed towards the modalities of sensible perception and their corporal codifications by the establishment of an anthropology of the senses.

Email : mlgelard@yahoo.fr

\section{Notes}

1. Pour un discours historique sur et à propos du Sahara, voir notamment Cuoq (1985) qui présente un corpus de sources sur les récits de voyageurs entre les VIIIe et XVIe siècles.

2. Je me permets de renvoyer à Gélard (2018).

3. En contexte berbérophone, peu d'études ont été consacrées à l'alimentation depuis les travaux pionniers de Gast (1968 et 2000).

4. Voir la description de la tribu des Ait Khebbach décrite plus loin.

5. Les habitants de Merzouga qui voyagent ou vivent à létranger soulignent souvent que la nourriture y est fade, sans saveur. « Tout a le même goût ! " disent-ils. Je reviendrai sur l'importance ontologique du goût face aux nourritures à destination des jnûns.

6. Le nom Amazigh (pluriel Imazighen) en langue tamazight est l'auto-désignation des populations dites «Berbères ». De nouvelles terminologies officielles ont été établies au Maroc par l'IRCAM (Institut Royal de la Culture Amazigh) qui utilise la forme latinisée "Amazighes » au lieu d'Imazighen. J’utilise pour ma part les dénominations usitées par les Aït Khebbach eux-mêmes, soit Imazighen.

7. Les Aït Khebbach se déclarent les «Ait Atta du soleil» (n-tafuyt), soit des oasis du Sud-Est par opposition aux « Ait Atta de l'ombre » (n-amalu) soit la chaîne montagneuse du Haut Atlas.

8. Situé en bordure de l'aire saharienne, au pied de l'erg Chebbi, le village de Merzouga fonctionne en économie oasienne. Lors de sa création, un imposant système d'irrigation (foggara) a dû être construit afin d'acheminer l'eau jusquà l'extrémité nord du village. L’eau, dont le débit est faible, permet toutefois d'irriguer la palmeraie (dattes et cultures vivrières).

9. Les familles font surtout du couscous le vendredi, jour de la prière.

10. Les plats de couscous sont échangés entre voisins et parents dans les différents quartiers du village. 
11. La région de Merzouga est très touristique, les hommes travaillent dans les auberges (nom donné aux installations hôtelières) alentour comme gérants, chameliers, guides ou cuisiniers. Lorsque l'on veut déconsidérer la cuisine d'une de ces auberges on fait alors référence à des " tagines piscine " c'est-à-dire sans goût car noyés d'eau!

12. Le mot est emprunté au français « corde » car la confection de tripes ressemble à la confection d'une corde.

13. Par exemple, lorsque la nourriture carnée est trop abondante.

14. En principe une année.

15. Je me permets de renvoyer à Gélard (2013).

16. Notons aussi l'importance de la cuisson par dessiccation (dans le sable), très fréquente en contexte nomade (Hincker 2005) aujourd'hui utilisée dans les bivouacs à l'intention des touristes.

17. Je n’entre pas ici dans le détail et les rituels nombreux qui entourent la sacralité de l'animal, voir notamment : Bonte et al. (1999), Brissebarre (1998) et Hammoudi (1988).

18. Comprendre : on va faire l'isgar, sous-entendu le rituel au sein duquel jeter (car c'est le sens même du mot isgar) signifie se débarrasser du mal.

19. A ma connaissance, le rituel d'isgar n’est réalisé qu'en milieu amazighophone.

20. Il s'agit du jour de la nomination des nourrissons, le septième jour après la naissance.

21. A. Epelboin indique la commercialisation depuis 2006 de papiers massicotés vendus en sac pour nourrir les moutons. Il existe également des accords entre les chauffeurs de camion des entreprises qui produisent beaucoup de déchets cartons et les éleveurs (information orale).

22. L'habitude de nourrir les animaux des noyaux de dattes se retrouve dans tout l'espace saharien (Jacques-Meunié 1982 et Gast 1968).

23. Je n'aborde ici que la consommation du lait animal. On sait l'importance du lait humain et son rôle dans l'univers des représentations de la parenté.

24. Le lait le plus apprécié est celui de chamelle, puis celui de chèvre parce que considérés comme les plus digestes. En contexte sédentaire, la consommation de lait a diminué considérablement en raison de la disparition de l'élevage des camélidés et des caprins.

25. D’un point de vue chimique, le colostrum n'a rien à voir avec la composition du lait mais, comme il apparaît avant celui-ci, il est souvent, en Occident et ailleurs, qualifié de " pré-lait », " premier lait».

26. Comme dans la plupart des sociétés déleveurs.

27. Je renvoie à l'ANR Colostrum, sous la direction de Joël Candau qui a bien souligné ces distinctions notables selon les pays où l'étude a été conduite. «Lalimentation pré-lactée (don et consommation néonatale du colostrum) : pratiques, représentations et enjeux de santé publique ». Dans le cadre de ce programme, une série d'entretiens réalisés en 2014 à Merzouga sont disponibles en ligne (à l'écoute et dans leur transcription) sur le site de la phonothèque d'Aix-en-Provence (http:// phonotheque.mmsh.univ-aix.fr/), dans le cadre du volet open science.

28. En arabe maghrébin on dit : «Où ta tête est-elle tombée (la première fois) ? " pour demander «Où es-tu né ?». 
29. Le colostrum cru n'est pas « mangeable » dit-on, il est impératif de le cuir.

30. Lallaitement a lieu en moyenne durant dix-huit mois.

31. La littérature à leur propos au Maghreb est fort vaste, je renvoie à quelques auteurs majeurs en contexte berbère : Camps-Fabrer 1998 ; Dermenghem 1954 ; Jacques-Meunié 1982.

32. D’une manière générale, le feu et la chaleur occupent une place prépondérante dans la médecine traditionnelle. Les brûlures au soufre ou au fer rouge sont très fréquentes pour soigner tout type de douleur. L'extrême chaleur attire le froid et soulage le malade. On dépose une petite quantité de soufre à l'endroit douloureux que l'on enflamme. La combustion sur la peau dure quelques minutes et forme alors une brûlure ronde qui, dit-on, va " aspirer le froid et l'eau ».

33. Le thé est parfumé d'un mélange d'herbes séchées qui proviennent toutes du désert.

34. Les frigidaires ne servent pas à cet usage, car leur température est estimée trop froide.

\section{Références citées}

Bonte, P. , Brissebarre, A.-M. et Gokalp, A. (1999), Sacrifices en islam : Espaces et temps d'un rituel (Paris : Editions du CNRS).

Brissebarre, A.-M. (2017), 'Lévolution de la pratique du sacrifice de l'aïd el-kebir en contexte urbain français', Ethnologie Française 168, no. 4 : 607-622.

Brissebarre, A.-M. (1998), La fête du mouton: Un sacrifice musulman dans l'espace urbain (Paris : Editions du CNRS).

Brisebarre, A.-M. et Kuczynski, L. (2009), La Tabaski au Sénégal : Une fête musulmane en milieu urbain (Paris : Karthala).

Camps-Fabrer, H. (1998), 'Génie', in Encyclopédie Berbère XX : 3023-3036.

Figueiredo, C. (2001), 'Conceptualisation des notions de chaud et de froid : Système d'éducation et relations hommes/femmes chez les Touaregs (Imédédaghen et Kel Adagh, Mali)' (Thèse de doctorat en ethnologie et anthropologie sociale, sous la direction de Pierre Bonte, Paris, EHESS).

Dermenghem, E. (1954), Le culte des saints dans l'Islam maghrébin (Paris : Gallimard).

Garreta, R. (2007), Des simples à l'essentiel : De l'herboristerie à l'aromathérapie, pratiques et représentations des plantes médicinales (Toulouse : Presses universitaires du Mirail).

Gast, M. (1968), Alimentation des populations de l'Ahaggar : Étude ethnographique (Paris : Arts et Métiers Graphiques).

Gast, M. (2000), Moissons du désert (Paris : Ibis Press).

Gélard, M.-L. (2018), 'Alimentation thérapeutique et rapports de séductions en contexte saharien (Sud-Est marocain) : la valorisation du sec', Journal des Africanistes 88, no. $1: 100-114$. 
Gélard, M.-L. (2016), 'Les nouvelles formes de médiation du patrimoine environnemental en contexte saharien : Les "bains de sable" ou l'immersion climatique, Ethnologies, Revue de l'Association canadienne d'ethnologie et de folklore 38, no. 1 : 171-193.

Gélard, M.-L. (2013), 'Les “bains de sable” dans le Tafilalt : Pratiques et représentations de l'immersion des corps en contexte saharien', Techniques \& Cultures 61 : 100-121.

Hammoudi, A. (1988), La victime et ses masques: Essai sur le sacrifice et la mascarade au Maghreb (Paris : Seuil).

Hincker, C. (2005), 'Le sec et l'humide : Appréciations touarègues des consistances alimentaires au Mali', Études Rurales 175-176 : 183-194.

Jacques-Meunié, D. (1982), Le Maroc saharien des origines à 1670, 2 tomes (Paris : Klincksieck).

Rachik, H. (1990), Sacré et sacrifice dans le Haut Atlas marocain (Casablanca : Afrique Orient).

Skounti, A. (2012), Le sang et le sol :. Nomadisme et sédentarisation au Maroc (Rabat : IRCAM).

Von Hoffmann, V. (2013), Goûter le monde : Une histoire culturelle du goût à l'époque moderne (Bruxelles : Peter Lang).

\section{Sites internet}

Climate-data.org : https//fr.climate-Data.org. Afrique, Maroc, Merzouga, consulté en janvier 2020.

Meteoblue, «Climat Merzouga» : www.meteoblue.com/fr/meteo/prevision/modelclimate/ merzouga_maroc_2542443, consulté en janvier 2020. 